Article

\title{
A Note on an Integral Transformation for the Equivalence between a Fractional and Integer Order Diffusion Model
}

\author{
Claudia A. Pérez-Pinacho ${ }^{*}+t^{+}$and Cristina Verde ${ }^{+}(D)$
}

check for updates

Citation: Pérez-Pinacho, C.A.; Verde, C. A Note on an Integral Transformation for the Equivalence between a Fractional and Integer Order Diffusion Model. Mathematics 2022, 10, 753. https://doi.org/ $10.3390 /$ math10050753

Received: 17 January 2022

Accepted: 23 February 2022

Published: 26 February 2022

Publisher's Note: MDPI stays neutral with regard to jurisdictional claims in published maps and institutional affiliations.

Copyright: (C) 2020 by the authors. Licensee MDPI, Basel, Switzerland. This article is an open access article distributed under the terms and conditions of the Creative Commons Attribution (CC BY) license (https:// creativecommons.org/licenses/by/ $4.0 /)$.
Instituto de Ingeniería, Universidad Nacional Autónoma de México, Mexico City 04510, Mexico; verde@unam.mx

* Correspondence: cperezp@iingen.unam.mx

+ These authors contributed equally to this work.

\begin{abstract}
This note tackles the equivalence problem between the fractional and integer order diffusion models. Unlike existing approaches, the existence of a unique integral transformation mapping the solution of the integer order model to a solution of the fractional order model of $\alpha=1 / 2$ is proven. Moreover, the corresponding inverse integral transformation is formally established to guarantee the equivalence and well-posedness of the solutions of these models. Finally, as an example, the solution of a fractional order diffusion model $\alpha=1 / 2$, obtained through the solution of its integer order counterpart and the proposed transformation, is compared with the solution derived by using the Fourier transform.
\end{abstract}

Keywords: integer order diffusion model; fractional calculus; integral transformation; fractional order diffusion model

MSC: 35R11; 35C15

\section{Introduction}

In recent years, fractional calculus has become an intensive field of study with diverse applications ranging from model-based fault detection [1-3], automatic control [4-8], the modeling of materials [9-11] up to the Susceptible-Infected-Recovered-Deceased (SIRD) model of COVID19 [12,13]. The prominent feature of fractional calculus in all applications is the use of the non-locality and memory effects of fractional order operators $[11,14]$ to describe physical phenomena associated with partial differential equations (PDE) [14-16]. Over the past few decades, two approaches have been developed by the scientific community for justifying the use of fractional calculus and for closely describing physical phenomena.

In the first one, by using the fractional order as an extra tuning parameter, some mathematical fractional order models are proposed based on matching experimental measurements of the output of a process with the output of a fractional order model $[10,17,18]$. Thus, whenever a fractional order has been found such that the outputs coincide, the tuned fractional order model is said to be an equivalent model for the process. This approach is well-known in the finite dimensional setting, and mathematical descriptions with a simple fractional order model have been exploited despite the lack of physical interpretation of the model and incompatibility of physical dimensions.

In the second one, inspired by the mathematical interest from the beginning of the fractional calculus, another approach consists of searching for mathematical models that contain some rational exponents in their transfer functions [17,19-22]. Because those exponents do not correspond to the effect described by derivatives and integrals in the classical sense, the mathematical models are analytically manipulated to obtain a fractional order model. In particular, it has been found that the study of physical phenomena described by PDE can be represented by non-locality and memory effects introduced 
by fractional order operators [14-16]. Some disadvantages of the PDE description are described below:

- Some approximations are performed in order to fulfill some definition of a fractional order operator $[15,17,19,21,22]$;

- There is no guarantee that the solution of the obtained fractional order model exists or how it is related to integer order PDE [20];

- No systematic method to obtain a fractional order model from an integer-order one exists.

In this framework, the work of Oldham and Spanier [15] glimpsed that a fractional diffusion model (FDM) can be derived from an integer order one by using ordinary calculus in the complex variable domain. Their finding was that the half-order diffusion model $D_{t}^{0.5} v(x, t)=-\frac{1}{\sqrt{b}} v_{x}(x, t)$ is embedded in the one-dimensional (1D) diffusion model (DM) $v_{t}(x, t)=\frac{1}{b} v_{x x}(x, t)$, where $\mathrm{b}$ is the diffusion's constant (see the details in Appendix A). This striking representation led to the study of physical phenomena described by diffusion models that can be represented by non-locality and memory effects introduced by derivatives and integrals of fractional order. It was indicated in [23] that the procedures [24-27] inspired by Oldham and Spanier's ideas do not attend to the following issues:

- The solution of the integer order DM should be explicitly known in the Laplace domain.

- The FDM is determined from an integer order model with zero initial conditions.

- The boundary conditions of FDM cannot be computed with existing methods from the boundary conditions of the integer order DM.

- The integer order DM cannot be obtained by only knowing the FDM and its solution.

Similar to the works in $[28,29]$ for the integer order PDE, a preliminary result in this direction was announced in the conference proceedings [23]. Specifically, the kernels of fractional order integral transformation and its inverse were presented, allowing to transform of the solution of the FDM with the Caputo derivative into the one-dimensional integer order diffusion model (1DDM) solution. The present note formally states the equivalence and well-posedness of the integer and fractional order diffusion models by means of the integral transformations proposed in [23]. Even though the results are correct, there were some inconsistencies in their formulation and proof. Specifically, this note corrects the assumptions used in [23], and the following details are introduced:

- Proposition 1 in [23] presented a unique transformation that maps the solution of the 1DDM to a solution for FDM. However, the main argument for demonstrating the uniqueness was based on undefined expressions obtained after applying the differentiation under the integral sign in a fractional order integral. To avoid the usage of undefined expressions, one can use the property to change the Caputo derivative to the Riemann-Liouville derivative of the integral term with a singular kernel.

- Proposition 2 in [23] provided that the solution of FDM maps to the solution of 1DDM by means of an inverse transformation. The formulation, however, was inaccurate and given without demonstration. The inverse fractional order integral transformation (IFOIT) in Proposition 2 must be called the right inverse of the fractional order integral transformation (FOIT) in Proposition 1 only when applied to the unique solution of the FDM with given boundary and initial conditions. Here, one formats Proposition 2 correctly, and the complete demonstration is given in detail.

- In the numerical example of [23], the obtained fractional order diffusion model and its solution are verified. The derivation of the boundary conditions missing in [23] is presented. Moreover, the solution of the fractional order diffusion model obtained by using the proposed transformation is validated by the Fourier transform method.

Thus, this note aims to clarify and improve the topics addressed in [1].

\section{Preliminaries}

In this section, the basic definitions and auxiliary results of partial fractional integrals and derivatives are summarized. 
Notation. $\mathbf{N}, \mathbb{R}, \mathbb{R}_{+}$denote the set of natural, real and positive real numbers, respectively. Let $[a, b] \subset \mathbb{R}$ and $[c, d] \subset \mathbb{R}$ denote compact intervals on the real line, and $g:[a, b] \rightarrow \mathbb{R}$ and $f:[a, b] \times[c, d] \rightarrow \mathbb{R}$ are real-valued functions of a single variable or two variables, respectively. $C^{1}[a, b]$ denotes the space of continuous differentiable functions on $[a, b]$ such that if $g \in C^{1}[a, b]$, then its derivative $g^{\prime}$ is continuous on $[a, b] . L_{1}[a, b]$ denotes the space of integrable functions over $[a, b]$, and $A C[a, b]$ denotes the space of absolutely continuous functions. Note that if $g \in A C[a, b]$, then $g$ has a derivative $g^{\prime}$ almost everywhere and $g^{\prime} \in L_{1}[a, b]$. Let $f(x, t)$ be a differentiable function on $(x, t) \in[a, b] \times[c, d]$, i.e., $f(\cdot, t) \in C^{1}[a, b]$ for all $x \in[a, b]$ and each $t \in[c, d]$, as well as $f(x, \cdot) \in C^{1}[a, b]$ for all $t \in[c, d]$ and each $x \in[a, b]$. The integer-order partial derivative of the function $f(x, t)$ with respect to the time $t$ and the spatial variable $x$ are denoted as $f_{t}(x, t):=\frac{\partial f(x, t)}{\partial t}$ and $f_{x}(x, t):=\frac{\partial f(x, t)}{\partial x}$, respectively. The symbol $\circ$ denotes the function composition. The symbol $\triangle$ terminates a claim. The following definitions and auxiliary results are immediate extensions of the results found in $[16,30,31]$.

\subsection{Definitions of Fractional Order Operators}

Definition 1. Ref. [30] Euler's Gamma function $\Gamma: \mathbb{R}_{+} \rightarrow \mathbb{R}_{+}$is defined by:

$$
\Gamma(z):=\int_{0}^{\infty} t^{z-1} e^{-t} d t
$$

Definition 2. Ref. [16] The Beta function is defined by the Euler integral of the first kind:

$$
B(y, p)=\int_{0}^{1} \xi^{y-1}(1-\xi)^{p-1} d \xi
$$

with $y, p \in \mathbb{R}_{+}$.

Definition 3. Ref. [16] Given $\ell, T \in \mathbb{R}_{+}$and with $f:[0, \ell] \times[0, T] \rightarrow \mathbb{R}, f(x, \cdot) \in L_{1}[0, T]$ for each $x \in[0, \ell]$, the Riemann-Liouville fractional integral (RLFI) of order a with respect to $t$ is defined by:

$$
I_{t}^{\alpha} f(x, t):=\frac{1}{\Gamma(\alpha)} \int_{0}^{t} f(x, \tau)(t-\tau)^{\alpha-1} d \tau,
$$

where $\Gamma(\cdot)$ denotes the Gamma function and $\alpha \in(0,1)$.

Definition 4. Ref. [16] Given $\ell, T \in \mathbb{R}_{+}$and with $f:[0, \ell] \times[0, T] \rightarrow \mathbb{R}, f(x, \cdot) \in L_{1}[0, T]$ for each $x \in[0, \ell]$, the Riemann-Liouville fractional partial derivative (RLFPD) of order $\alpha$ with respect to $t$ is defined by:

$$
\mathfrak{D}_{t}^{\alpha} f(x, t):=\frac{\partial}{\partial t}\left[\frac{1}{\Gamma(1-\alpha)} \int_{0}^{t} f(x, \tau)(t-\tau)^{-\alpha} d \tau\right]
$$

where $\alpha \in(0,1)$.

Definition 5. Ref. [16] Given $\ell, T \in \mathbb{R}_{+}$and with $f:[0, \ell] \times[0, T] \rightarrow \mathbb{R}, f(x, \cdot) \in A C[0, T]$ for each $x \in[0, \ell]$, the Caputo fractional partial derivative (CFPD) of order $\alpha$ with respect to $t$ is defined by:

$$
D_{t}^{\alpha} f(x, t):=\frac{1}{\Gamma(1-\alpha)} \int_{0}^{t} \frac{f_{\tau}(x, \tau)}{(t-\tau)^{\alpha}} d \tau,
$$

where $\Gamma(\cdot)$ denotes the Gamma function and $\alpha \in(0,1)$.

\subsection{Properties of Fractional Order Operators}

Property 1. Ref. [16] The Beta function satisfies:

$$
B(y, p)=\frac{\Gamma(p) \Gamma(y)}{\Gamma(y+p)},
$$


where $\Gamma(\cdot)$ denotes the Gamma function.

Property 2. Ref. [31] Let $\ell, T \in \mathbb{R}_{+}$, if $f(x, \cdot) \in A C[0, T]$ for each $x \in[0, \ell]$. Thus:

$$
\lim _{\alpha \rightarrow 1} D_{t}^{\alpha} f(x, t)=f_{t}(x, t) \text {. }
$$

Property 3. According to [30], the CFPD satisfies the following semigroup property. Let $\ell, T \in \mathbb{R}_{+}$, consider $f(x, \cdot) \in C^{1}[0, T]$ for almost all $x \in[0, \ell]$, and let $\alpha, \beta>0$ be such that $[\alpha, \alpha+\beta] \in[0,1]$, resulting in:

$$
D_{t}^{\alpha} D_{t}^{\beta} f(x, t)=D_{t}^{\beta+\alpha} f(x, t) .
$$

Property 4. Theorem 3.7 in [30]. Let $\ell, T \in \mathbb{R}_{+}$and $(x, t) \in[0, \ell] \times[0, T]$. If $f(x, \cdot) \in C[0, T]$, and $\alpha \geq 0$ for each $x \in[0, \ell]$, then:

$$
D_{t}^{\alpha} I_{t}^{\alpha} f(x, t)=f(x, t)
$$

By a generalization of the fundamental theorem of calculus, the CFPD can be understood as the left inverse of the RLFI operator. The CFPD, however, is not the right inverse for the RLFI as the following property indicates.

Property 5. Theorem 3.8 in [30]. Let $(x, t) \in[0, \ell] \times[0, T]$. If $f(x, \cdot) \in A C[0, T], \alpha \in(0,1)$ for each $x \in[0, \ell]$, then:

$$
I_{t}^{\alpha} D_{t}^{\alpha} f(x, t)=f(x, t)-f(x, 0) .
$$

Property 6. Lemma 3.5 in [30]. Let $(x, t) \in[0, \ell] \times[0, T]$ and $\alpha \in(0,1)$. Assume that $\Delta(x, t)$ is such that both $D_{t}^{\alpha} \Delta(x, t)$ and $\mathfrak{D}_{t}^{\alpha} \Delta(x, t)$ exist. Thus:

$$
\mathfrak{D}_{t}^{\alpha} \Delta(x, t)=D_{t}^{\alpha} \Delta(x, t)
$$

if and only if $\Delta(x, 0)=0$.

Property 7. Lemma 2.24 in [16]. Let $\ell, T \in \mathbb{R}_{+}, \alpha \in(0,1), f:[0, \ell] \times[0, T] \rightarrow \mathbb{R}$, if $f(x, \cdot) \in$ $L_{1}[0, T]$ and $f(x, \cdot) \in A C[0, T]$ for each $x \in[0, \ell)$, then the Laplace transform of the CFPD is given by:

$$
\mathcal{L}\left\{D_{t}^{\alpha} f(x, t)\right\}=s^{\alpha} F(x, s)-s^{\alpha-1} f(x, 0),
$$

where $F(x, s)=\int_{0}^{\infty} f(x, t) \mathrm{e}^{-s t} d t$ is the Laplace transform with respect to time of $f(x, t)$ for all $x \in[0, \ell]$.

\section{Equivalence between 1DDM and FDM}

Consider the nondimensional 1DDM:

$$
v_{x x}(x, t)=v_{t}(x, t)
$$

and let $\phi:[0, \ell] \rightarrow \mathbb{R}$ and $\rho:[0, T] \rightarrow \mathbb{R}$ be functions satisfying the boundary and initial conditions:

$$
\begin{aligned}
& v(x, 0)=\phi(x), \\
& v(0, t)=\rho(t), \\
& v(\ell, t)<\infty
\end{aligned}
$$

such that for all $(x, t) \in[0, \ell] \times[0, T]$, the solution $v(x, t)$ of $(12)$ exists and is unique. The following claims summarized the declarations given in [23]:

Claim 1. The solution $w(x, t)$ of the FDM of order $\alpha=1 / 2$ is: 


$$
D_{t}^{\alpha} w(x, t)=-w_{x}(x, t),
$$

with the initial and boundary conditions given by:

$$
\begin{aligned}
& w(x, 0)=\phi(x), \\
& w(0, t)=\rho(t)-I_{t}^{\alpha} v_{x}(0, t), \\
& w(\ell, t)<\infty
\end{aligned}
$$

on the same bounded domain of (12) can be expressed as the fractional order integral transformation (FOIT):

$$
w(x, t)=v(x, t)-\int_{0}^{t} k(t, \tau) v_{x}(x, \tau) d \tau,
$$

with kernel function:

$$
k(t, \tau)=\frac{1}{\sqrt{\pi}} \frac{1}{(t-\tau)^{\alpha}}
$$

and $v(x, t)$ solution of (12) and (13).

Claim 2. The solution of $v(x, t)$ of 1DDM (12) with initial and boundary conditions in (13) can be represented as the inverse fractional order integral transformation (IFOIT):

$$
v(x, t)=w(x, t)+\frac{1}{2 \sqrt{\pi}} \int_{0}^{t} \frac{1}{(t-\tau)^{1 / 2}} w_{x}(x, \tau) d \tau
$$

with $w(x, t)$ solution of (14) and (15).

Claim 3. IFOIT (18) is the right inverse of the FOIT (16).

As mentioned in the introduction, the proof of Claim 1 involved undefined terms. Claim 2 was not demonstrated before, and in Claim 3, important considerations about the unique solution of the FDM with boundary and initial conditions were missing. Thus, the following section shows how to eliminate these issues.

\section{Precise Formulation of Claims with Proof}

The following two propositions, corresponding to Claims 1 through 3 and establishing the equivalence between the models, are formulated precisely from those given in [23].

\subsection{Direct Integral Transformation}

The next result is given to guarantee that the solution of (12) maps to the solution of (14) in Claim 1.

Proposition 1. Let $v(x, t)$ be the solution of the 1DDM (12) on the bounded domain $(x, t) \in$ $[0, \ell] \times[0, T]$ with initial and boundary conditions given by (13) and consider that kernel function $k(t)$ and $v_{x}(x, t)$ are $\mathcal{L}_{1}[0, T]$ functions for each $x$. Therefore, the FOIT (16) is the unique solution of (14) on the same bounded domain of (12) with its boundary and initial conditions given by (15), if and only if the kernel function is $k(t-\tau)=\frac{1}{\sqrt{\pi}} \frac{1}{(t-\tau)^{\alpha}}$, and the fractional order is $\alpha=\frac{1}{2}$.

Proof. Necessary condition: To determine the unique transformation, it is necessary to replace (16) in the target system (14) and find $k(t, \tau)$ and $\alpha$ as follows. 
By taking the derivative of the transformation (16) with respect to $x$ and by using the model (12) with the integration by parts of the last term, one can write:

$$
\begin{aligned}
w_{x}(x, t) & =v_{x}(x, t)-\int_{0}^{t} k(t, \tau) v_{x x}(x, \tau) d \tau, \\
& =v_{x}(x, t)-\int_{0}^{t} k(t, \tau) v_{\tau}(x, \tau) d \tau .
\end{aligned}
$$

By using Property 4, the first term of (19) can be written as:

$$
v_{x}(x, t)=D_{t}^{\alpha} I_{t}^{\alpha} v_{x}(x, t),
$$

Set $\Theta(x, t) \triangleq I_{t}^{\alpha} v_{x}(x, t)$, through Property 6 and the RLFPD in Definition 4 , then:

$$
\begin{aligned}
v_{x}(x, t) & =D_{t}^{\alpha} \Theta(x, t)=\mathfrak{D}_{t}^{\alpha} \Theta(x, t) \\
& =\frac{\partial}{\partial t}\left[\frac{1}{\Gamma(1-\alpha)} \int_{0}^{t} \Theta(x, \tau)(t-\tau)^{-\alpha} d \tau\right],
\end{aligned}
$$

Therefore, the right-hand side of the target system (14) is given by:

$$
w_{x}(x, t)=\frac{\partial}{\partial t}\left[\frac{1}{\Gamma(1-\alpha)} \int_{0}^{t} \Theta(x, \tau)(t-\tau)^{-\alpha} d \tau\right]-\int_{0}^{t} k(t, \tau) v_{\tau}(x, \tau) d \tau .
$$

On the other hand, according to Definition 5 , the CFPD of $w(x, t)$ can be written as:

$$
\begin{aligned}
D_{t}^{\alpha} w(x, t) & =\frac{1}{\Gamma(1-\alpha)} \int_{0}^{t}(t-\tau)^{-\alpha} \frac{\partial}{\partial \tau}\left[v(x, \tau)-\int_{0}^{\tau} k(\tau, \xi) v_{x}(x, \xi) d \xi\right] d \tau \\
& =\frac{1}{\Gamma(1-\alpha)} \int_{0}^{t}(t-\tau)^{-\alpha} v_{\tau}(x, \tau) d \tau-D_{t}^{\alpha} \varphi(x, t)
\end{aligned}
$$

where $\varphi(x, t) \triangleq \int_{0}^{\tau} k(t, \xi) v_{x}(x, \xi) d \xi$. By Property 6 , the second term with the CFPD can be rewritten according to an RLFPD as:

$$
\begin{aligned}
D_{t}^{\alpha} \varphi(x, t) & =\mathfrak{D}_{t}^{\alpha} \varphi(x, t) \\
& =\frac{\partial}{\partial t}\left[\frac{1}{\Gamma(1-\alpha)} \int_{0}^{t} \varphi(x, \tau)(t-\tau)^{-\alpha} d \tau\right],
\end{aligned}
$$

The left-hand side of the target system (14) is given by:

$$
\begin{aligned}
D_{t}^{\alpha} w(x, t) & =\frac{1}{\Gamma(1-\alpha)} \int_{0}^{t}(t-\tau)^{-\alpha} v_{\tau}(x, \tau) d \tau \\
& -\frac{\partial}{\partial t}\left[\frac{1}{\Gamma(1-\alpha)} \int_{0}^{t} \varphi(x, \tau)(t-\tau)^{-\alpha} d \tau\right],
\end{aligned}
$$

Finally, by adding (22) and (25), one can write:

$$
\begin{aligned}
w_{x}(x, t) & +D_{t}^{\alpha} w(x, t)=\frac{\partial}{\partial t}\left[\frac{1}{\Gamma(1-\alpha)} \int_{0}^{t} \Theta(x, \tau)(t-\tau)^{-\alpha} d \tau\right] \\
& -\int_{0}^{t} k(t, \tau) v_{\tau}(x, \tau) d \tau+\frac{1}{\Gamma(1-\alpha)} \int_{0}^{t}(t-\tau)^{-\alpha} v_{\tau}(x, \tau) d \tau \\
& -\frac{\partial}{\partial t}\left[\frac{1}{\Gamma(1-\alpha)} \int_{0}^{t} \varphi(x, \tau)(t-\tau)^{-\alpha} d \tau\right] .
\end{aligned}
$$

By factorizing similar terms of the right-hand side of (26), one obtains: 


$$
\begin{aligned}
w_{x}(x, t) & +D_{t}^{\alpha} w(x, t)=\frac{\partial}{\partial t}\left(\frac{1}{\Gamma(1-\alpha)} \int_{0}^{t}(t-\tau)^{-\alpha}[\Theta(x, \tau)-\varphi(x, \tau)]\right) d \tau \\
& +\int_{0}^{t} \Omega(t, \tau) v_{\tau}(x, \tau) d \tau
\end{aligned}
$$

where

$$
\Theta(x, \tau)-\varphi(x, \tau)=\int_{0}^{\tau} v_{x}(x, \xi)\left[\frac{1}{\Gamma(\alpha)(t-\xi)^{1-\alpha}}-k(t, \xi)\right] d \xi
$$

and

$$
\Omega(t, \tau)=\frac{(t-\tau)^{-\alpha}}{\Gamma(1-\alpha)}-k(t, \tau) .
$$

Therefore, $w(x, t)$ in (27) is a solution of (14) only if the right-hand side of (27) is equal to 0 for $v(x, t)$ solution of (12) and (13) on its bounded domain. Henceforth, from Titchmarsh convolution theorem in [32], Equations (28) and (29) must be 0. As a consequence, the following two conditions must be satisfied:

Condition 1: $k(\tau, \xi)=\frac{1}{\Gamma(\alpha)(\tau-\xi)^{1-\alpha}} ;$

Condition 2: $k(t, \tau)=\frac{1}{\Gamma(1-\alpha)(t-\tau)^{\alpha}}$.

Thus, it remains to obtain the function $k(t-\tau)$ and the parameter $\alpha$ such that Condition 1 implies Condition 2. A dummy change of variables in Condition 1 and Condition 2 yields:

$$
k(t, \tau)=\frac{1}{\Gamma(\alpha)(t-\tau)^{1-\alpha}}=\frac{1}{\Gamma(1-\alpha)(t-\tau)^{\alpha}},
$$

which can be rewritten as:

$$
\frac{\Gamma(\alpha)}{(t-\tau)^{\alpha}}=\frac{\Gamma(1-\alpha)}{(t-\tau)^{1-\alpha}} .
$$

Thus, this equality holds when $\alpha=1-\alpha$, i.e., $\alpha=1 / 2$.

In summary, if $\alpha=1 / 2$, the kernel function $k(t)=\frac{1}{\Gamma\left(\frac{1}{2}\right)(t)^{\frac{1}{2}}}=\frac{1}{\sqrt{\pi t}}$ is assigned to the direct integral transformation:

$$
w(x, t)=v(x, t)-\frac{1}{\sqrt{\pi}} \int_{0}^{t} \frac{1}{(t-\tau)^{1 / 2}} v_{x}(x, \tau) d \tau,
$$

then, the fractional diffusion model:

$$
D_{t}^{1 / 2} w(x, t)=-w_{x}(x, t)
$$

is obtained.

Since $v_{x}(x, t)$ and $k(t, \tau)$ are $\mathcal{L}_{1}[0, T]$ functions (note for $k(t, \tau)$ that $\frac{1}{\sqrt{\pi}} \int_{0}^{T}\left|t^{-\frac{1}{2}}\right| d t=$ $\left.-\frac{2}{\sqrt{\pi}} \sqrt{T}<\infty\right)$, the integral term in (31) is well-defined for all fixed $x \in[0, \ell]$, and the initial and boundary conditions of (32) can be fixed by evaluating the integral transformation (31). Thus, one obtains the conditions:

- $\quad$ For $t=0, w(x, 0)=v(x, 0)=\phi(x)$;

- $\quad$ For $x=0, w(0, t)=\rho(t)-\left.\frac{1}{\sqrt{\pi}} \int_{0}^{t} \frac{1}{(t-\tau)^{1 / 2}} v_{x}(x, \tau)\right|_{x=0} d \tau$;

- $\quad$ For $x=\ell<\infty, w(0, t)=v(\ell, t)-\left.\frac{1}{\sqrt{\pi}} \int_{0}^{t} \frac{1}{(t-\tau)^{1 / 2}} v_{x}(x, \tau)\right|_{x=\ell} d \tau<\infty$. 
Sufficient condition: This condition is trivial, and it follows by substituting the FOIT (16) with $\alpha=1 / 2$ and $k(t, \tau)=\frac{1}{\sqrt{\pi}(t-\tau)^{1 / 2}}$ into the 1DDM in (12), which yields to FDM (14).

\subsection{Inverse Integral Transformation}

For Claims 2 and 3, to guarantee the equivalent relation between (12) and (32), the transformation such that the solution of (12) can be expressed by the inverse of (31) is presented in the following sufficient condition.

Proposition 2. Let $w(x, t)$ be the solution of the FDM (32) on the bounded domain $(x, t) \in$ $[0, \ell] \times[0, T]$ with initial and boundary conditions given by $(15)$ and $w_{x}(x, t)$ be $\mathcal{L}_{1}[0, T]$ function for all $x$. If the inverse integral transformation is given by (18), then the IFOIT (18) is the right inverse of the FOIT (31) with $w(x, t)$ solution of (32), subject to conditions (15). Moreover, $v(x, t)$ is the solution of the 1DDM (12) on the same bounded domain and conditions given by (13).

Proof. The proof consists of two steps. First, by substituting (18) into (31), it is shown that the IFOIT (18) is the right inverse of the FOIT (31). Second, the IFOIT (18) is substituted into 1DDM (12) to obtain the FDM (32).

Step 1. Substituting the IFOIT (18) into FOIT (31) yields:

$$
\begin{aligned}
w(x, t) & =w(x, t)+\frac{1}{2 \sqrt{\pi}} \int_{0}^{t} \frac{w_{x}(x, \tau)}{(t-\tau)^{1 / 2}} d \tau \\
& -\frac{1}{\sqrt{\pi}} \int_{0}^{t} \frac{1}{(t-\tau)^{1 / 2}}\left(w_{x}(x, \tau)+\frac{1}{2 \sqrt{\pi}} \int_{0}^{\tau} \frac{w_{x x}(x, \xi)}{(t-\xi)^{1 / 2}} d \xi\right) d \tau,
\end{aligned}
$$

By using (32) and Properties 2 and 3, one can see that:

$$
\begin{aligned}
w_{x}(x, t) & =-D_{t}^{1 / 2} w(x, t) \\
w_{x x}(x, t) & =\frac{\partial}{\partial x} \underbrace{w_{x}(x, t)}_{-D_{t}^{1 / 2} w(x, t)}=-D_{t}^{1 / 2} \underbrace{w_{x}(x, t)}_{-D_{t}^{1 / 2} w(x, t)}=w_{t}(x, t) .
\end{aligned}
$$

By substituting (34) and (35) into (33), it follows that:

$$
\begin{aligned}
w(x, t) & =w(x, t)-\frac{1}{2 \sqrt{\pi}} \int_{0}^{t} \frac{D_{\tau}^{1 / 2} w(x, \tau)}{(t-\tau)^{1 / 2}} d \tau+\frac{1}{\sqrt{\pi}} \int_{0}^{t} \frac{D_{\tau}^{1 / 2} w(x, \tau)}{(t-\tau)^{1 / 2}} d \tau \\
& -\frac{1}{2 \sqrt{\pi}} \int_{0}^{t} \frac{1}{(t-\tau)^{1 / 2}}\left(\frac{1}{\sqrt{\pi}} \int_{0}^{\tau} \frac{w_{\xi}(x, \xi)}{(t-\xi)^{1 / 2}} d \xi\right) d \tau \\
& =w(x, t)-\frac{1}{2 \sqrt{\pi}} \int_{0}^{t} \frac{D_{\tau}^{1 / 2} w(x, \tau)}{(t-\tau)^{1 / 2}} d \tau+\frac{1}{\sqrt{\pi}} \int_{0}^{t} \frac{D_{\tau}^{1 / 2} w(x, \tau)}{(t-\tau)^{1 / 2}} d \tau \\
& -\frac{1}{2 \sqrt{\pi}} \int_{0}^{t} \frac{1}{(t-\tau)^{1 / 2}} D_{\tau}^{1 / 2} w(x, \tau) d \tau,
\end{aligned}
$$

and by using RLFI (2) for $\alpha=1 / 2$, one can write:

$$
\begin{aligned}
w(x, t) & =w(x, t)-\frac{1}{2} I_{t}^{1 / 2} D_{t}^{1 / 2} w(x, t)+I_{t}^{1 / 2} D_{t}^{1 / 2} w(x, t)-\frac{1}{2} I_{t}^{1 / 2} D_{t}^{1 / 2} w(x, t) \\
& =w(x, t) .
\end{aligned}
$$

Let $v:=T_{\operatorname{inv}}(w)$ and $w:=T_{\operatorname{dir}}(v)$ denote the transformations in (18) and (31), respectively. Since the equality $w(x, t)=T_{\text {dir }} \circ T_{\text {inv }}(w(x, t))=w(x, t)$ in (37) is obtained, then $T_{\mathrm{inv}}(w)$ is the right inverse for $T_{\mathrm{dir}}(v)$. 
Step 2. By using the inverse transformation (18), it is possible to return to the original model (12) from (32). For this purpose, differentiate (18) once with respect to $t$ and twice with respect to $x$, as indicated by (12). To achieve this, differentiating the inverse transformation (18) with respect to $t$ yields:

$$
v_{t}(x, t)=w_{t}(x, t)+\frac{1}{2 \sqrt{\pi}} \frac{\partial}{\partial t} \int_{0}^{t} \frac{1}{(t-\tau)^{1 / 2}} w_{x}(x, \tau) d \tau .
$$

By substituting (32) into (37) and using Property 5, the right-hand side of (12) can than be written as follows:

$$
\begin{aligned}
v_{t}(x, t) & =w_{t}(x, t)-\frac{1}{2} \frac{\partial}{\partial t} I_{t}^{1 / 2} D_{t}^{1 / 2} w(x, t) \\
& =w_{t}(x, t)-\frac{1}{2} \frac{\partial}{\partial t}(w(x, t)-w(x, 0)) \\
& =w_{t}(x, t)-\frac{1}{2} \frac{\partial}{\partial t}(w(x, t)-\phi(x)) \\
& =w_{t}(x, t)-\frac{1}{2} w_{t}(x, t)
\end{aligned}
$$

Note that in (38) the fact that $\frac{\partial}{\partial t} \phi(x)=0$ was used. On the other hand, differentiating (18) twice with respect to $x$, one obtains:

$$
\begin{aligned}
v_{x x}(x, t) & =w_{x x}(x, t)+\frac{1}{2 \sqrt{\pi}} \frac{\partial}{\partial x} \int_{0}^{t} \frac{w_{x x}(x, \tau)}{(t-\tau)^{1 / 2}} d \tau \\
& =w_{t}(x, t)+\frac{1}{2 \sqrt{\pi}} \frac{\partial}{\partial x} D_{t}^{1 / 2} w(x, t) \\
& =w_{t}(x, t)-\frac{1}{2} D_{t}^{1 / 2} D_{t}^{1 / 2} w(x, t),
\end{aligned}
$$

and the aid of Property 3, the left-hand side of (12) can be written as follows:

$$
v_{x x}(x, t)=w_{t}(x, t)-\frac{1}{2} w_{t}(x, t) .
$$

By subtracting (39) from (38), the following 1DDM is reached (12):

$$
v_{t}(x, t)-v_{x x}(x, t)=w_{t}(x, t)-\frac{1}{2} w_{t}(x, t)-w_{t}(x, t)+\frac{1}{2} w_{t}(x, t)=0 .
$$

Finally, because (18) is the right inverse of the integral transformation (31), the boundary and initial conditions (13) are simply obtained by substituting them (15) into the inverse transformation (18).

\section{Example for Specific Boundary Conditions}

This section shows the FDM solution obtained in the example of [23] is the correct one. Consider the case of the 1DDM (12) with the following boundary and initial conditions $[23,33]$ :

$$
\begin{aligned}
& v(x, 0)=0 \\
& v(0, t)=u(t) \\
& v(\ell, t)<\infty,
\end{aligned}
$$


where $u(t)$ is the Heaviside step function. By considering Proposition 1, the solution of the target system (32) exists and is unique. In addition, the boundary and initial conditions:

$$
\begin{aligned}
& w(x, 0)=0, \\
& w(0, t)=2 u(t), \\
& w(\ell, t)<\infty,
\end{aligned}
$$

are obtained from the transformation (31) (see Appendix B).

As derived in [23], the solution of the FDM (32) can be obtained through the direct FOIT (31) by considering the solution of the 1DDM (12), $v(x, t)=\operatorname{erfc}\left(\frac{x}{2 \sqrt{t}}\right)$, satisfying the boundary and initial conditions (40). After applying the direct FOIT (31), one obtains the solution of the FDM given by:

$$
w(x, t)=2 \operatorname{erfc}\left(\frac{x}{2 \sqrt{t}}\right) .
$$

Indeed, by using the Fourier transform with respect to $x$ in both sides of (32), one obtains:

$$
-i \omega \bar{W}(\omega, t)+\bar{W}(0, t)=D_{t}^{\frac{1}{2}} \bar{W}(\omega, t)
$$

Then, one can write:

$$
-i \omega \bar{W}(\omega, s)+\bar{W}(0, s)=s^{\frac{1}{2}} \bar{W}(\omega, s)-s^{\frac{1}{2}} \bar{W}^{\prime}(\omega, 0),
$$

by considering the boundary condition in the Laplace domain $W(0, s)=2 / s$, the above equation is reduced to:

$$
\begin{aligned}
-i \omega \bar{W}(\omega, s)+\frac{2}{s} & =s^{\frac{1}{2}} \bar{W}(\omega, s) \\
\bar{W}(\omega, s) & =\frac{2}{s\left(s^{\frac{1}{2}}+i \omega\right)}
\end{aligned}
$$

Thus, the inverse Fourier transform of (43) is given by:

$$
W(x, s)=\frac{2}{s} e^{-\sqrt{s} x},
$$

which corresponds to the Laplace transform of the function given in (42).

\section{Conclusions}

This note discussed and formalized the new insight into the fractional order integral transformation for the diffusion model. In particular, adequate assumptions are proposed to complete and clarify the results given in [23]. This contribution is devoted to studying the equivalence between systems modeled with integer order and fractional order operators, specifically of order $\alpha=1 / 2$. The analysis centers on the one-dimensional diffusion equation and establishes equivalence through integral transformations between the integer and fractional order model. It is validated that the relationship between the integer model and the fractional model of diffusion already exists in the literature; nevertheless, the methodology commonly found is formed by manipulating the solution of the integer order model in the Laplace domain to derive a fractional order model from which it is not possible to recover the integer model. This is not the case proposed in this paper, which is based on a bidirectional link between the two models through the integral transformations. It is worth mentioning that the integral transformations are not general; they are restricted to different orders and definitions of derivatives and integrals according to the models under study. This opens the way, however, for new research opportunities: In the physical 
application's viewpoint, the work opens new horizons for treating diffusive problems in some contexts where fractional calculus has been necessary. In particular, the results may be helpful in studying some aspects of the anomalous diffusion phenomenon.

Author Contributions: Conceptualization, C.A.P.-P. and C.V.; Formal analysis, C.A.P.-P. and C.V.; Investigation, C.A.P.-P.; Methodology, C.A.P.-P. and C.V.; Supervision, C.V.; Writing-review-editing, C.A.P.-P. and C.V. All authors have read and agreed to the published version of the manuscript.

Funding: The authors acknowledge the financial support from the DGAPA-UNAM posdoctoral Fellowship, IT100122-DGAPA-UNAM and the Conacyt 2021-2022 posdoctoral.

Institutional Review Board Statement: Not applicable.

Informed Consent Statement: Not applicable.

Data Availability Statement: Not applicable.

Conflicts of Interest: The authors declare no conflict of interest.

\section{Appendix A. Classical Derivation of the Fractional Order Diffusion Model}

Consider the 1DDM:

$$
v_{x x}(x, t)=b v_{t}(x, t),
$$

where $b>0$ denotes the diffusion constant and $v:[0, L] \times[0, T] \rightarrow \mathbb{R}$ denotes the density of the diffusion material. Let $v(0, t)=u(t)$, and let $v(\ell, t)<\infty$ and $v(x, 0)=0$ be the boundary and initial conditions for Equation (A1). It is easy to see that the solution of Equation (A1) in the Laplace domain yields:

$$
V(x, s)=V(0, s) \exp (-x \sqrt{b s}) .
$$

It is possible to obtain a fractional order operator in the Laplace domain by differentiating Equation (A2) with respect to $x$ such that:

$$
V_{x}(x, s)=-\sqrt{b s} V(x, s) .
$$

From the Laplace inversion of Equation (A3) and the definition of CFPD given in (4) with the initial condition $v(x, 0)=0$, the following fractional equation is obtained

$$
D_{t}^{1 / 2} v(x, t)=-\frac{1}{\sqrt{b}} v_{x}(x, t) .
$$

Notice that for this procedure, one must know beforehand the solution of system in the Laplace domain in Equation (A2), the specific boundary conditions, and a restriction in the initial condition in order to fulfill a definition of the derivative. Moreover, boundary and initial conditions for Equation (A4) are clearly not the same as those given for Equation (A1). In fact, those are not possible to determine once the differentiation of Equation (A2) takes place. 


\section{Appendix B. A Boundary Condition}

The solution of the diffusion model (12) is $v(x, t)=\operatorname{erfc}(z)$, where $z=\frac{x}{2} \sqrt{\frac{1}{t}}$. By applying the transformation (31) to the boundary condition $v(0, t)$ in $(40)$, one obtains:

$$
\begin{aligned}
w(0, t) & =v(0, t)-\left.\frac{1}{\sqrt{\pi}} \int_{0}^{t} \frac{1}{(t-\tau)^{1 / 2}} v_{x}(x, \tau)\right|_{x=0} d \tau \\
& =u(t)-\left.\frac{1}{\sqrt{\pi}} \int_{0}^{t} \frac{1}{(t-\tau)^{1 / 2}}\left(\frac{-e^{-z^{2}}}{\sqrt{\pi \tau}}\right)\right|_{x=0} d \tau \\
& =u(t)-\underbrace{\frac{1}{\sqrt{\pi}} \int_{0}^{t} \frac{1}{(t-\tau)^{1 / 2}} \frac{1}{\sqrt{\pi \tau}}}_{\text {RLFI }} d \tau .
\end{aligned}
$$

The RLFI in Equation (A5) can be solved by a change in variable $\tau=\xi t$ and $d \tau=t d \xi$ with $\tau \in[0, t]$ and $\xi \in[0,1]$, respectively. Thus:

$$
\frac{1}{\pi} \int_{0}^{1} \frac{1}{\sqrt{\xi t}(t-\xi t)^{1 / 2}} t d \xi=\frac{1}{\pi} \int_{0}^{1} \xi^{-1 / 2}(1-\xi)^{-1 / 2} d \xi,
$$

and note that if

$$
p-1=-1 / 2 \Leftrightarrow p=1 / 2, y-1=-1 / 2 \Leftrightarrow y=1 / 2,
$$

for the Beta function in (1), then:

$$
\frac{1}{\pi} \int_{0}^{1} \quad \xi^{-1 / 2}(1-\xi)^{-1 / 2} d \xi=\frac{B\left(\frac{1}{2}, \frac{1}{2}\right)}{\pi} .
$$

From Property 1 , it follows that $B\left(\frac{1}{2}, \frac{1}{2}\right) / \pi=1 / \Gamma(1)=u(t)$.

\section{References}

1. Kopka, R. Model-Based Fault Diagnosis with Fractional Models. In Advances in Modelling and Control of Non-Integer-Order Systems; Springer: Berlin/Heidelberg, Germany, 2015; pp. 257-263.

2. Azimi, M.; Shandiz, H.T. Simultaneous Fault Detection and Control Design for Linear Fractional-Order Systems. Iran. J. Sci. Technol. Trans. Electr. Eng. 2020, 44, 485-494. [CrossRef]

3. He, Y.; Fu, Y.; Qiao, Z.; Kang, Y. Chaotic resonance in a fractional-order oscillator system with application to mechanical fault diagnosis. Chaos Solitons Fractals 2021, 142, 110536. [CrossRef]

4. Monje, C.A.; Chen, Y.; Vinagre, B.M.; Xue, D.; Feliu-Batlle, V. Fractional-Order Systems and Controls: Fundamentals and Applications; Springer Science \& Business Media: Berlin/Heidelberg, Germany, 2010.

5. Caponetto, R.; Dongola, G.; Fortuna, L.; Petras, I. Fractional Order Systems: Modeling and Control Applications; World Scientific: Singapore, 2010; Volume 72.

6. Modiri, A.; Mobayen, S. Adaptive terminal sliding mode control scheme for synchronization of fractional-order uncertain chaotic systems. ISA Trans. 2020, 105, 33-50. [CrossRef] [PubMed]

7. Oustaloup, A. On the CRONE Control. In Diversity and Non-Integer Differentiation for System Dynamics; Wiley: Hoboken, NJ, USA, 2014; pp. 139-164.

8. Jajarmi, A.; Baleanu, D. On the fractional optimal control problems with a general derivative operator. Asian J. Control 2021, 23, 1062-1071. [CrossRef]

9. Bonfanti, A.; Kaplan, J.L.; Charras, G.; Kabla, A. Fractional viscoelastic models for power-law materials. Soft Matter 2020, 16, 6002-6020. [CrossRef] [PubMed]

10. Biswas, K.; Bohannan, G.; Caponetto, R.; Lopes, A.M.; Machado, J.A.T. Fractional-Order Devices; Springer: Berlin/Heidelberg, Germany, 2017.

11. Ortigueira, M.D. Fractional Calculus for Scientists and Engineers; Springer Science \& Business Media: Berlin/Heidelberg, Germany, 2011; Volume 84.

12. Nisar, K.S.; Ahmad, S.; Ullah, A.; Shah, K.; Alrabaiah, H.; Arfan, M. Mathematical analysis of SIRD model of COVID-19 with Caputo fractional derivative based on real data. Results Phys. 2021, 21, 103772. [CrossRef] [PubMed] 
13. Jahanshahi, H.; Munoz-Pacheco, J.M.; Bekiros, S.; Alotaibi, N.D. A fractional-order SIRD model with time-dependent memory indexes for encompassing the multi-fractional characteristics of the COVID-19. Chaos Solitons Fractals 2021, 143, 110632. [CrossRef] [PubMed]

14. Podlubny, I. Fractional Differential Equations: An Introduction to Fractional Derivatives, Fractional Differential Equations, to Methods of Their Solution and Some of Their Applications; Elsevier: Amsterdam, The Netherlands, 1998; Volume 198.

15. Oldham, K.; Spanier, J. The Fractional Calculus Theory and Applications of Differentiation and Integration to Arbitrary Order; Elsevier: Amsterdam, The Netherlands, 1974; Volume 111.

16. Kilbas, A.A.A.; Srivastava, H.M.; Trujillo, J.J. Theory and Applications of Fractional Differential Equations; Elsevier Science Limited: Amsterdam, The Netherlands, 2006; Volume 204.

17. Radwan, A.G.; Salama, K.N. Fractional-order RC and RL circuits. Circuits Syst. Signal Process. 2012, 31, 1901-1915. [CrossRef]

18. Sabatier, J.; Agrawal, O.P.; Machado, J.T. Advances in Fractional Calculus; Springer: Berlin/Heidelberg, Germany, 2007 ; Volume 4.

19. Carlson, G.; Halijak, C. Approximation of fractional capacitors $(1 / \mathrm{s})^{\wedge}(1 / \mathrm{n})$ by a regular Newton process. IEEE Trans. Circuit Theory 1964, 11, 210-213. [CrossRef]

20. Nigmatullin, R. The realization of the generalized transfer equation in a medium with fractal geometry. Phys. Status Solidi B 1986, 133, 425-430. [CrossRef]

21. Machado, J. Discrete-time fractional-order controllers. Fract. Calc. Appl. Anal. 2001, 4, 47-66.

22. Valsa, J.; Vlach, J. RC models of a constant phase element. Int. J. Circuit Theory Appl. 2013, 41, 59-67. [CrossRef]

23. Pérez-Pinacho, C.A.; Verde, C. A fractional-order integral transformation for the diffusion model. In Proceedings of the 2021 Congreso Nacional de Control Automático, CNCA 2021, Guanajuato, Mexico, 13-15 October 2021; pp. 127-133.

24. Metzler, R.; Glöckle, W.G.; Nonnenmacher, T.F. Fractional model equation for anomalous diffusion. Phys. A Stat. Mech. Its Appl. 1994, 211, 13-24. [CrossRef]

25. Zhang, Y.; Xue, D. Modeling and simulating transmission lines using fractional calculus. In Proceedings of the 2007 International Conference on Wireless Communications, Networking and Mobile Computing, IEEE, Shanghai, China, 21-25 September 2007; pp. 3115-3118.

26. Kulish, V.V.; Lage, J.L. Application of fractional calculus to fluid mechanics. J. Fluids Eng. 2002, 124, 803-806. [CrossRef]

27. Sierociuk, D.; Dzieliński, A.; Sarwas, G.; Petras, I.; Podlubny, I.; Skovranek, T. Modelling heat transfer in heterogeneous media using fractional calculus. Philos. Trans. R. Soc. A Math. Phys. Eng. Sci. 2013, 371, 20120146. [CrossRef] [PubMed]

28. Colton, D. The solution of initial-boundary value problems for parabolic equations by the method of integral operators. J. Differ Equ. 1977, 26, 181-190. [CrossRef]

29. Seidman, T.I. Two results on exact boundary control of parabolic equations. Appl. Math. Optim. 1984, 11, 145-152. [CrossRef]

30. Diethelm, K. The Analysis of Fractional Differential Equations: An Application-Oriented Exposition Using Differential Operators of Caputo Type; Springer Science \& Business Media: Berlin/Heidelberg, Germany, 2010.

31. Ishteva, M. Properties and Applications of the Caputo Fractional Operator; Department of Mathematics, University of Karlsruhe: Karlsruhe, Germany , 2005.

32. Titchmarsh, E.C. The zeros of certain integral functions. Proc. Lond. Math. Soc. 1926, 2, 283-302. [CrossRef]

33. Cheng, D.K. Analysis of Linear Systems; Addison-Wesley: Boston, MA, USA, 1959. 